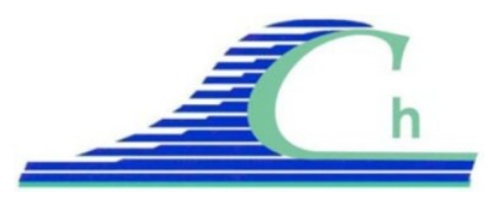

XII ${ }^{\text {èmes }}$ Journées Nationales Génie Côtier - Génie Civil

Cherbourg, 12-14 juin 2012

DOI:10.5150/jngcgc.2012.044-S ～C Editions Paralia CFL

disponible en ligne - http://www.paralia.fr - available online

\title{
Vers une description paramétrée du taux de dépôt effectif des sédiments cohésifs
}

\section{Martin SANCHEZ ${ }^{1}$}

\author{
1. Université de Nantes, LPGN, UMR 6112 CNRS, BP 92208, 44322 Nantes, France. \\ martin.sanchez@univ-nantes.fr
}

\section{Résumé :}

Le taux de dépôt effectif des sédiments cohésifs qui est usuellement décrit par le modèle de Krone est évalué dans cette étude par une méthode originale alternative grâce à la description simultanée de trois processus sédimentaires : (i) le dépôt des matières en suspension, (ii) le tassement des vases déposées et (iii) la remise en suspension des matériaux préalablement sédimentés. Une application est effectuée prenant comme référence la vase de l'estuaire de la Loire.

L'approche développée ne fait pas explicitement appel au concept de valeur seuil $\tau_{d}$ de la contrainte hydrodynamique au fond $\tau_{o}$ au-dessous de laquelle les sédiments se déposent, mais les résultats obtenus permettent d'effectuer une analyse des facteurs contrôlant la valeur de $\tau_{d}$.

\section{Mots-clés :}

Taux de dépôt - Taux d’érosion - Sédiment cohésif - Seuil de dépôt - Tassement Vase de Loire

\section{Introduction}

Dans les zones côtières et estuariennes, les deux principaux agents hydrodynamiques sont le courant et la houle. Les théories actuelles de transport sédimentaire caractérisent la "force des agents hydrodynamiques" par la contrainte de cisaillement au fond $\tau_{o}$.

Les expérimentations effectuées par plusieurs auteurs (MEHTA, 1986) montrent que le dépôt de sédiments fins en suspension ne peut se produire que pour des conditions proches de celles d'eau calme ( $\tau_{o}$ proche de zéro). Le modèle le plus utilisé pour évaluer le taux de dépôt effectif $D^{e f}$ est celui de Krone qui s’écrit comme suit (KRONE, 1986 ; MEHTA, 1986) :

$$
D^{e f}=\left\{\begin{array}{lll}
\overline{W_{o}} C_{o}\left(1-\tau_{o} / \tau_{d}\right) & \text {, si : } & \tau_{o}<\tau_{d} \\
0 & \text {, si : } & \tau_{o} \geq \tau_{d}
\end{array}\right.
$$

où $\tau_{d}$ est la valeur de $\tau_{o}$ au-dessus de laquelle les sédiments ne se déposent plus, $\overline{W_{o}} C_{o}=D$ définit le flux de sédiment vers le fond, le terme $\left(1-\tau_{o} / \tau_{d}\right)$ représente selon Krone la probabilité que les agrégats touchant le fond adhérent à celui-ci, et enfin, le produit $\overline{W_{o}}\left(1-\tau_{o} / \tau_{d}\right)$ définit une vitesse de chute moyenne apparente des sédiments près du fond. 
Les valeurs de $\tau_{d}$ utilisées par divers auteurs pour différents sédiments varient entre $0,04 \mathrm{~N} \mathrm{~m}^{-2}$ et 0,20 $\mathrm{N} \mathrm{m}^{-2}$ (MEHTA, 1986 ; KRISHNAPPAN \& ENGEL, 1994).

L'objectif de cette étude est d'explorer la possibilité de modéliser le taux de dépôt effectif $D^{e f}$ à travers la description simultanée de 3 différents processus sédimentaires régissant la dynamique verticale des sédiments cohésifs. L'application de cette approche concerne la vase de Loire pour laquelle on connaît les valeurs des différents paramètres physiques concernés.

\section{Description du transport en suspension de sédiments}

Les sédiments peuvent être transportés en suspension par les courants à la même vitesse horizontale que ceux-ci. Dans un écoulement turbulent, la valeur instantanée de la concentration $c$ en matières en suspension oscille autour d'une valeur moyenne $C$. La décomposition de $c$ s'écrit comme suit :

$c=C+c^{\prime}$

où $c^{\prime}$ est la composante fluctuante de la concentration. Si la diffusion moléculaire des sédiments en suspension est négligée, la concentration $C$ dans un écoulement turbulent à surface libre unidirectionnel (suivant $O x$ ) est régie par l'équation de conservation suivante :

$\frac{\partial C}{\partial t}+V_{x} \frac{\partial C}{\partial x}=-\frac{\partial\left\langle v_{x}{ }^{\prime} c^{\prime}\right\rangle}{\partial x}-\frac{\partial\left\langle v_{y}{ }^{\prime} c^{\prime}\right\rangle}{\partial y}-\frac{\partial\left\langle v_{z}{ }^{\prime} c^{\prime}\right\rangle}{\partial z}+\frac{\partial(\bar{W} C)}{\partial z}$

où $\bar{W}$ est la vitesse de chute moyenne des matières en suspension, et les termes $\left\langle v_{x}{ }^{\prime} c^{\prime}\right\rangle$, $\left\langle v_{y}{ }^{\prime} c^{\prime}\right\rangle,\left\langle v_{z}{ }^{\prime} c^{\prime}\right\rangle$, représentent les corrélations temporelles entre les composantes de la vitesse fluctuante et la concentration fluctuante ; ils traduisent le transfert de masse lié à la turbulence. D’habitude ces termes sont modélisés à travers les lois de diffusion de Fick-Boussinesq suivantes :

$$
\left\langle v_{x}{ }^{\prime} C^{\prime}\right\rangle=-K_{x} \frac{\partial C}{\partial x},\left\langle v_{y}{ }^{\prime} c^{\prime}\right\rangle=-K_{y} \frac{\partial C}{\partial y},\left\langle v_{z}{ }^{\prime} c^{\prime}\right\rangle=-K_{z} \frac{\partial C}{\partial z}
$$

où $K_{x}, K_{y}$ et $K_{z}$ sont les coefficients de diffusion turbulente de masse selon les directions $O x, O y$ et $O z$ respectivement. L'équation de conservation devient :

$$
\frac{\partial C}{\partial t}+V_{x} \frac{\partial C}{\partial x}=\frac{\partial}{\partial x}\left(K_{x} \frac{\partial C}{\partial x}\right)+\frac{\partial}{\partial y}\left(K_{y} \frac{\partial C}{\partial y}\right)+\frac{\partial}{\partial z}\left(K_{z} \frac{\partial C}{\partial z}\right)+\frac{\partial(\bar{W} C)}{\partial z}
$$

La solution de cette équation doit satisfaire à une condition de frontière relative aux échanges de sédiments entre l'écoulement et le fond (dépôts et érosions). Par la suite, dans cette section, on considère un transport sans érosion et sans dépôt.

Dans un écoulement et un régime sédimentaire stationnaires et uniformes, si la diffusion transversale (suivant $O y$ ) de sédiments est nulle, la distribution verticale des sédiments obéit à l'équation suivante :

$$
\bar{W} C=-K_{z} \frac{\partial C}{\partial z}
$$




\section{XII ${ }^{\text {èmes }}$ Journées Nationales Génie Côtier - Génie Civil \\ Cherbourg, 12-14 juin 2012}

Si le fond se situe en $z=0$, l'intégration de cette équation permet d'écrire :

$C(z)=C_{o} \exp \left(-\int_{a}^{z} \frac{\bar{W}}{K_{z}} \mathrm{~d} z\right)$

où $C_{0}$ est une concentration de référence au niveau $z=a$ très proche du fond.

L'équation précédente admet des solutions analytiques dépendant des expressions de $\bar{W}$ et de $K_{\mathrm{z}}$. La plus connue de ces solutions, due à ROUSE (1937), est obtenue en prenant comme constante la valeur de $\bar{W}$ avec le coefficient de diffusion turbulente $K_{z}$ égal au coefficient de viscosité turbulente $v_{T}$, lequel est modélisé par l'expression suivante :

$K_{z} \approx \kappa u_{*} z\left(1-\frac{z}{d}\right)$

où $\kappa \approx 0,4$ est la constante universelle de Karman, $u *$ la vitesse de cisaillement et $d$ la profondeur La distribution verticale de $C(z)$ obéit alors à la loi de Rouse :

$C(z)=C_{o}\left[\frac{a(d-z)}{z(d-a)}\right]^{\bar{W} /\left(\kappa u_{*}\right)}$

D'autres solutions de l'équation 7 existent pour une vitesse de chute considérée comme une variable stochastique (SANCHEZ, 2006) correspondant à la présence simultanée en suspension de particules de sédiment avec des vitesses de chute variables selon leur taille et leur densité.

\subsection{Transport en suspension des sédiments non cohésifs en régime stationnaire}

Bien que les modes de transport les plus connus pour les sables soient par charriage des couches superficielles des matériaux déposés et par saltation des grains, ces sédiments peuvent éventuellement être transportés en suspension si leur vitesse de chute est inférieure à environ 0,8 fois la vitesse de cisaillement $u *$ (TEETER, 1986). Pour le calcul du débit solide longitudinal associé à ce mode de transport on admet que la distribution verticale des matières en suspension s'adapte à chaque instant aux actions hydrodynamiques (régime stationnaire graduellement varié).

Selon les approches actuelles, la distribution des matières en suspension résulte d'un équilibre au fond (en $z=a$ ) entre un flux d'érosion $E$ et un flux de dépôt $D$. En régime stationnaire le profil vertical de $C$ est donné par l'équation 7 , avec $C_{o}$ qui dépend de la condition limite au fond qui s'écrit :

Flux vertical au fond $=-\left.K_{z} \frac{\partial C}{\partial z}\right|_{z=a}-\overline{W_{o}} C_{o}=E-D=0$

où $\overline{W_{o}}$ désigne la vitesse de chute moyenne des sédiments du fond. Toujours en régime stationnaire dans le cas des sables, le flux de dépôt est considéré être égal à $\overline{W_{o}} C_{o}$. Alors, la concentration de référence au fond $C_{o}$ est reliée à $E$ par :

$E=\overline{W_{o}} C_{o}$

Ainsi, le profil vertical de $C$ obéit à : 


$$
C(z)=\frac{E}{\overline{W_{o}}} \exp \left(-\int_{a}^{z} \frac{\bar{W}}{K_{z}} \mathrm{~d} z\right)
$$

Pour un sédiment donné, une loi empirique relie le flux d'érosion $E$ et les conditions hydrodynamiques ("pick-up function"). Il est intéressant de noter que le régime stationnaire pour le transport en suspension de sédiments non cohésifs correspond à un équilibre entre le flux de dépôt $D=\overline{W_{o}} C_{o}$ (chute des particules sous l'effet du poids propre) et le flux d'érosion $E$ (remise en suspension des sédiments déposés sous l'effet de forces turbulentes de l'écoulement). Il est entendu que les particules qui se déposent ne sont pas exactement les mêmes que celles qui se mettent en suspension.

\subsection{Transport en suspension des sédiments cohésifs en régime stationnaire}

Un profil stationnaire de la concentration en matières en suspension peut également exister dans le cas du transport des sédiments cohésifs. La condition d'équilibre correspond toujours à une égalité entre les flux de dépôt et d'érosion, mais cette fois-ci les particules touchant le fond entraînées par le flux de dépôt n’adhérent pas aux sédiments préalablement déposés, de sorte que les particules déposées et érodées peuvent être exactement les mêmes.

La condition d'équilibre peut se présenter quand tous les sédiments déposés susceptibles d'être remis en suspension par un écoulement l'ont été et par la suite les conditions hydrodynamiques sont restées stationnaires pendant un laps de temps suffisant pour parvenir à un équilibre dans la distribution des matières en suspension dans la colonne d'eau.

Ainsi par exemple, si l'on considère l'action prolongée d'un écoulement érosif, l'eau va se charger progressivement jusqu'à l'érosion complète des sédiments déposés susceptibles d'être remis en suspension par cet écoulement. Bien que très variable dans le temps et dans l'espace, la quantité des sédiments érodables est en général limitée. En effet, la quantité de ces sédiments dépend des érosions et dépôts préalables et du temps de tassement qui contrôle la concentration dans les couches de vase déposées et par conséquent la résistance à l'érosion.

L'étude de la dynamique des sédiments cohésifs dans un environnement côtier nécessite la mise au point d'un modèle de transport hydrodynamique couplé à un modèle de transport sédimentaire tenant compte des principaux processus sédimentaires qui sont : (i) le dépôt des matières en suspension, (ii) le tassement des vases déposées et (iii) la remise en suspension des matériaux du fond. Ces processus seront décrits dans la section suivante de cette note.

En toute rigueur, pour les matériaux cohésifs on devrait distinguer deux flux d'érosion : a)un flux d'érosion $E^{\prime}$ concernant les particules touchant le fond entraînées par le flux de dépôt $\left(0<E^{\prime}<\overline{W_{o}} C_{o}\right)$; le taux de dépôt effectif est alors :

$D^{e f}=D-E^{\prime}=\overline{W_{o}} C_{o}-E^{\prime}$. 


\section{XII ${ }^{\text {èmes }}$ Journées Nationales Génie Côtier - Génie Civil \\ Cherbourg, 12-14 juin 2012}

b)un flux d'érosion effectif $E^{e f}$ concernant les sédiments préalablement déposés; la valeur de $E^{e f}$ dépend des caractéristiques physiques des sédiments déposés et des forces érosives.

\section{Description des processus sédimentaires affectant les sédiments cohésifs}

\subsection{Vitesse de chute des sédiments}

La vitesse de chute de sédiments cohésifs en suspension dans les cours d'eau est très supérieure à celle des grains d'argile individuels. Ceci s'explique par le fait que la plus grande partie de la masse sédimentaire en suspension se rassemble sous la forme d'agrégats. La vitesse de chute synthétise certaines caractéristiques de ces agrégats telles que : la taille, la forme et la masse volumique.

La vitesse de chute varie principalement en fonction de la concentration locale $C$ (KRONE, 1986 ; MIGNIOT, 1989). Pour les suspensions diluées la vitesse de chute augmente avec $C$. Pour les suspensions très concentrées $\left(C>\sim 10 \mathrm{~kg} \mathrm{~m}^{-3}\right)$ la vitesse de chute diminue quand $C$ augmente (vitesse entravée, MEHTA, 1986). La variation de la vitesse de chute moyenne avec la concentration $C$ dans les suspensions diluées est souvent décrite par la loi de Owen suivante :

$\bar{W}=m C^{n}$

où $m$ et $n$ sont deux constantes propres à chaque sédiment. Les valeurs de l'exposant $n$ varient de 0,5 à 2 selon les auteurs et les valeurs de $m$ sont telles que le maximum de $\bar{W}$ avant la chute entravée est de l'ordre de $0,5 \mathrm{à} 4 \mathrm{~mm} \mathrm{~s}^{-1}$, selon les sédiments. Les valeurs retenues dans cette étude sont : $n=0,50$ et $m=0,001$ (unités S.I.).

\subsection{Tassement des dépôts de crème de vase}

Si l'on admet que les contraintes effectives sont nulles pendant les processus de tassement de la crème de vase, on obtient l'expression de BEEN (1980) valable pour la vitesse de tassement (entravée) :

$V=\frac{k}{\rho_{w}}\left(1-\frac{\rho_{w}}{\rho_{s}}\right) C$

où $k$ est le coefficient de perméabilité qui dépend exclusivement de la concentration $C$, $\rho_{s}$ la masse volumique de la phase solide $\left(\rho_{s} \approx 2550 \mathrm{~kg} \mathrm{~m}^{-3}\right)$ et $\rho_{w}$ la masse volumique de l'eau. Pour un grand nombre de sédiments cohésifs, la variation de $k$ avec $C$ peut être décrite par (SANCHEZ \& LEVACHER, 2007) :

$k=A_{1} \exp \left[-A_{2}\left(\frac{C}{\rho_{s}}\right)^{A_{3}}\right]$

où $A_{1}\left(\mathrm{~m} \mathrm{~s}^{-1}\right), A_{2}$ et $A_{3}$ sont des constantes spécifiques à chaque sédiment. 
La figure 1 montre la variation des valeurs de $k$ en fonction de $C$ évaluées à partir de mesures en laboratoire effectuées par plusieurs auteurs sur des vases de Loire. L'ajustement de l'équation 15 pour ces sédiments a permis d'évaluer $A_{1}=1,60 \mathrm{~m} \mathrm{~s}^{-1}$, $A_{2}=72$ et $A_{3}=0,65$, valables pour la crème de vase de Loire dans la gamme de concentrations définie par $15 \mathrm{~kg} \mathrm{~m}^{-3}<C<325 \mathrm{~kg} \mathrm{~m}^{-3}$.

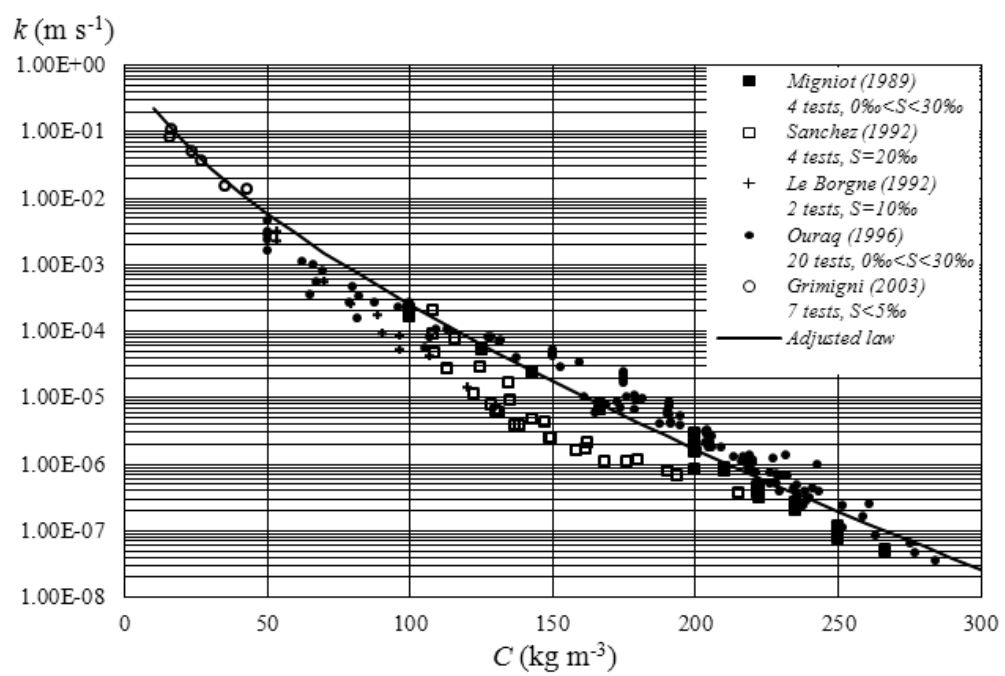

Figure 1. Coefficient de perméabilité $k$ en fonction de la concentration $C$ pour les dépôts de vase de l'estuaire de la Loire (SANCHEZ\& LEVACHER, 2007).

Un modèle de tassement de crème de vase basé sur la théorie de sédimentation de KYNCH (1952) modifiée par BEEN (1980) a été mis au point par SANCHEZ et GROVEL (1993). Selon ce modèle, le flux solide à travers l'interface mobile de concentration $C$ constante dans le temps (c'est-à-dire, la quantité de masse solide qui excède la concentration $C$ par unité de surface horizontale et par unité de temps dans la couche de crème de vase) est donné par l'équation suivante (voir figure 2) :

$Q_{C}=V C\left[A_{2} A_{3}\left(\frac{C}{\rho_{s}}\right)^{A_{3}}-1\right]$

Pendant le dépôt des sédiments en suspension, une discontinuité dans le profil de la concentration peut apparaitre à l'interface entre une couche de sédiments déposés et l'écoulement au-dessus de cette couche. La concentration $C_{S}$ à la surface de la couche de sédiments déposés dépend de la valeur du flux de dépôt $D$. Cette concentration $C_{S}$ est donnée par l'abscisse associée à une valeur de l'ordonnée égale à $D$ par le biais de la courbe de $Q_{C}$ montrée sur la figure 2 . Si l'hypothèse d'un milieu continu est dûment justifiée, trois cas peuvent se présenter :

a) si le flux de dépôt $D$ est inférieur à $7.4 \times 10^{-6} \mathrm{~kg} \mathrm{~m}^{-2} \mathrm{~s}^{-1}$, les sédiments se déposent avec une concentration en surface supérieure à $325 \mathrm{~kg} \mathrm{~m}^{-3}$ (il n’y a pas de formation de crème de vase dans ce cas) ; 
b) si $D$ varie entre $7.4 \times 10^{-6}$ et $2.6 \times 10^{-2} \mathrm{~kg} \mathrm{~m}^{-2} \mathrm{~s}^{-1}$, une crème de vase avec une

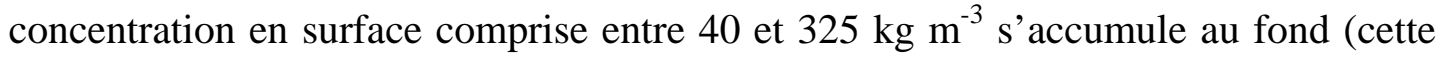
couche de dépôt comporte un gradient de concentration vertical) ;

c) si $D$ est supérieur à $2.6 \times 10^{-2} \mathrm{~kg} \mathrm{~m}^{-2} \mathrm{~s}^{-1}$, les nouveaux sédiments déposés s'accumulent au fond avec une concentration initiale de $40 \mathrm{~kg} \mathrm{~m}^{-3}$.

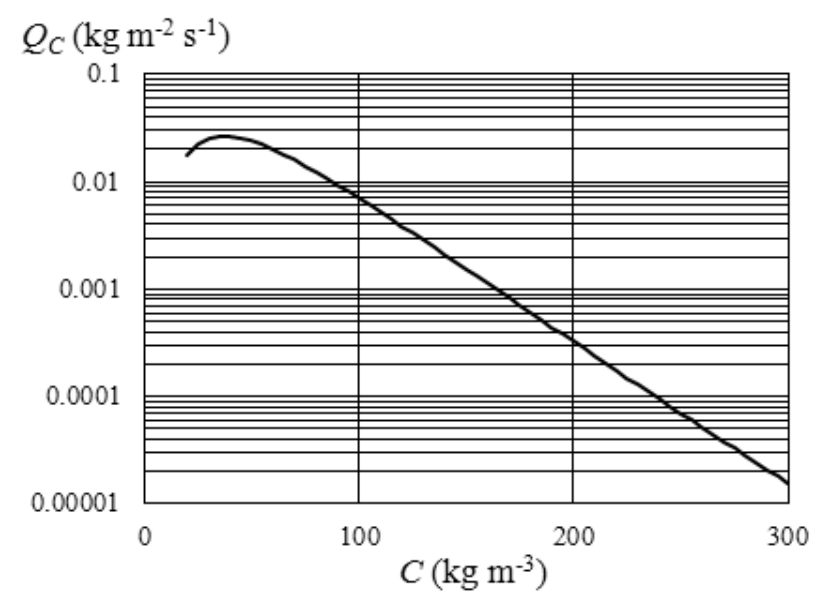

Figure 2. Flux solide $Q_{C}$ à travers une interface de concentration $C$ constant dans le temps. Cette courbe a été établie pour les dépôts de vase de l'estuaire de la Loire.

\subsection{Flux d'érosion effectif}

Une étude de l'érosion de la crème de vase effectuée à l'université de Nantes (HOSSEINI, 1999; SANCHEZ \& LEVACHER, 2008) a permis d'établir une expression reliant la contrainte tangentielle critique d'érosion $\tau_{e}$ aux paramètres caractéristiques du sédiment déposé :

$$
\tau_{e}=0,294 \tau_{y}\left(\frac{\rho-\rho_{w}}{\rho_{w}}\right)^{1,11} \quad \text { si: } \quad C<420 \mathrm{kgm}^{-3}
$$

où $\rho$ est la masse volumique de la vase déposée et $\tau_{y}$ la rigidité initiale de cette vase. Selon cette loi, le début de remise en suspension correspond à une vitesse d'érosion résiduelle $E_{v o}=3 \times 10^{-7} \mathrm{~m} \mathrm{~s}^{-1}$ (équivalent à l'érosion d'une couche de $1 \times 10^{-3} \mathrm{~m}$ d'épaisseur en une heure). La vitesse d'érosion est décrite par :

$$
E_{v}=\left\{\begin{array}{lll}
E_{v o}\left(\tau_{o} / \tau_{e}\right)^{\alpha}, \text { si }: & \tau_{o}<\tau_{e} \\
E_{v o} \exp \left[\alpha\left(\tau_{o} / \tau_{e}-1\right)\right], \text { si }: & \tau_{o} \geq \tau_{e}
\end{array}\right.
$$

Ainsi, pour $\tau_{0}<\tau_{e}$ la vitesse d'érosion est calculée par une loi semblable à celle utilisée par DELO et OCKENDEN (1992), et par CERCO et al. (2000). Pour $\tau_{0} \geq \tau_{c e}$ l'expression retenue a été proposée par MEHTA et PARTHENIADES (1982). Le paramètre $\alpha$ est lié à $\tau_{e}$ par :

$\alpha=0,1194 \frac{\sqrt{\tau_{e} / \rho_{w}} \times \varepsilon}{v}$ 
où $v$ est la viscosité cinématique de l'eau et $\varepsilon$ la rugosité du dépôt. D’après les travaux de HOSSEINI (1999) nous avons retenu pour la vase de Loire $\varepsilon=8,7 \times 10^{-4} \mathrm{~m}$. Pendant ces travaux, la mesure de la rigidité initiale avec un rhéomètre Brookfield LVT a permis de rattacher $\tau_{y}$ à $C$ par :

$$
\tau_{y}=\left\{\begin{array}{lll}
1.39 \times 10^{-5} C^{2.36} & \text {, si: } & 40 \mathrm{~kg} \mathrm{~m}^{-3}<C<182 \mathrm{~kg} \mathrm{~m}^{-3} \\
1.50 \times 10^{-11} C^{5} & \text {, si: } & 182 \mathrm{~kg} \mathrm{~m}^{-3}<C<420 \mathrm{~kg} \mathrm{~m}^{-3}
\end{array}\right.
$$

Le flux d'érosion effectif $E^{e f}$ (quantité de masse sèche remise en suspension par unité de surface horizontale et par unité de temps) est donné par $E_{v}$ multiplié par la concentration $C_{S}$ sur la surface de la couche de vase déposée.

\section{Résultats de la modélisation du taux de dépôt effectif}

Dans cette étude, le taux de dépôt effectif de la vase de Loire est calculé suivant une démarche originale, différente de celle de Krone (équation 1). Ce taux est évalué à travers une modélisation simultanée des processus de dépôt, de tassement et de remise en suspension provoquée par une action hydrodynamique.

Les forces d'adhérence entre les agrégats de vase touchant le fond et les sédiments préalablement déposés, sont paramétrées comme des rigidités initiales d'un milieu continu équivalent qui pourraient être mesurées avec un rhéomètre Brookfield LVT.

Si les agrégats en cours de dépôt tombent sur une couche de crème de vase avec une faible rigidité initiale $\left(\tau_{y}<3 \mathrm{~N} \mathrm{~m}^{-2}\right)$ ou si le flux de dépôt est suffisamment élevé pour engendrer la formation d'une telle couche de crème de vase, les sédiments en cours de dépôt sont considérés comme faisant partie d'un milieu continu incluant les sédiments préalablement déposés. Dans ce cas, la rigidité initiale des agrégats touchant le fond est paramétrée égale à celle associée à la valeur de $C_{S}$ à travers l'équation 20.

Si le flux de dépôt est suffisamment faible et les agrégats se déposant touchent un fond de moyenne ou de forte rigidité initiale $\left(\tau_{y}>3 \mathrm{~N} \mathrm{~m}^{-2}\right)$, ces agrégats ne peuvent pas être assimilés comme faisant partie du milieu continu constitué par les sédiments préalablement déposés. Les agrégats déposés restent individualisés touchant les sédiments sous-jacents sur une surface de contact. Selon les travaux de KRONE (1986), la structure de ce type de surface de contact est d'un ordre d'agrégation supérieur à celles des agrégats (structure plus lâche), de sorte que la résistance au cisaillement de cette surface est d'un ordre de grandeur inférieur à celle des agrégats.

Dans cette étude, la rigidité initiale équivalente de ces surfaces de contact est paramétrée égale à $3 \mathrm{~N} \mathrm{~m}^{-2}$ (cette valeur définit la frontière entre une valeur faible et moyenne de la rigidité initiale selon l'équation 20). Cette rigidité initiale correspond à une concentration $C_{S \max }=182 \mathrm{~kg} \mathrm{~m}^{-3}$. Ainsi, dans l'algorithme du modèle du taux de dépôt effectif, cette dernière valeur de la concentration est retenue comme la valeur initiale maximale possible de la concentration en surface résultant d'un flux de dépôt.

La modélisation comporte les étapes suivantes : 


\section{XII ${ }^{\text {èmes }}$ Journées Nationales Génie Côtier - Génie Civil \\ Cherbourg, 12-14 juin 2012}

a) pour une valeur donnée de $C_{o}$, la vitesse de chute moyenne $\overline{W_{o}}$ des sédiments proches du fond est calculée par l'équation 13, de sorte que le flux de dépôt peut être évalué par $\overline{W_{o}} C_{o}$;

b) la concentration à la surface du dépôt $C_{S} \leq C_{S \max }$ est calculée à l'aide du modèle de tassement en fonction du flux de dépôt $\overline{W_{o}} C_{o}$ et du tassement de la couche de vase déposée sous-jacente (voir section 3.2) ;

c) pour chaque valeur donnée de $\tau_{o}$, le flux d'érosion $E^{\prime}=\operatorname{Min}\left[E_{v} C_{S} ; \overline{W_{o}} C_{o}\right]$ des sédiments juste déposés est calculé à l'aide de l'équation 18 ;

d) le taux de dépôt effectif est évalué par $D^{e f}=\overline{W_{o}} C_{o}-E^{\prime}$;

e) si $D^{e f}=0$, un éventuel taux d'érosion effectif peut être calculé en fonction de la contrainte hydrodynamique et de la concentration de la crème de vase sous-jacente.

De fait, le taux de dépôt effectif est ainsi considéré dépendre du degré de tassement de la couche de sédiments préalablement déposés. Dans ce qui suit, le taux de dépôt est étudié pour deux cas susceptibles d'être observés.

\subsection{Dépôt au-dessus d'une crème de vase avec une concentration préalable élevée}

Ce cas peut se présenter quand les vitesses du courant sont en train de ralentir. La concentration en surface de la couche de vase préalablement déposée est supérieure à celle résultant du flux de dépôt $\overline{W_{o}} C_{o}$. Les sédiments se déposant ont une concentration en surface $C_{S} \leq C_{S \max }$ dépendant de $\overline{W_{o}} C_{o}$. En effet, la valeur de $C_{S}$ est obtenue à partir de la courbe de $Q_{C}$ montrée sur la figure 2 (voir section 3.2). La figure 3 montre le taux de dépôt effectif réduit $D^{e f} /\left(\overline{W_{o}} C_{o}\right)$ en fonction de $\tau_{o}$, calculé pour six valeurs de la concentration $C_{o}$ en matières en suspension près du fond. Le tableau 1 récapitule plusieurs paramètres propres à cette modélisation.

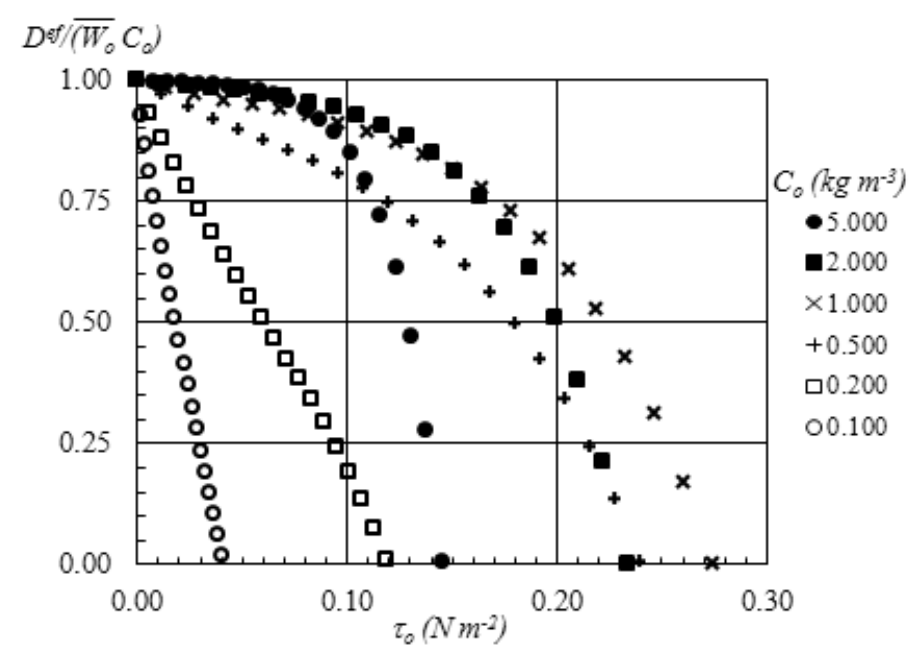

Figure 3. Valeurs de $D^{e f} /\left(\overline{W_{o}} C_{o}\right)$ simulées en fonction de $\tau_{o}$ pour un dépôt au-dessus d'une couche de crème de vase ayant une concentration préalable élevée. 
Tableau 1. Paramètres de la modélisation de $D^{e f}$ pour une concentration élevée de la vase préalablement déposée.

\begin{tabular}{lllll}
\hline$C_{o}\left(\mathrm{~kg} \mathrm{~m}^{-3}\right)$ & $\overline{W_{o}}\left(\mathrm{~m} \mathrm{~s}^{-1}\right)$ & $C_{o} \times \overline{W_{o}}\left(\mathrm{~kg} \mathrm{~m}^{-2} \mathrm{~s}^{-1}\right)$ & $C_{S}\left(\mathrm{~kg} \mathrm{~m}^{-3}\right)$ & $\tau_{e}\left(\mathrm{~N} \mathrm{~m}^{-2}\right)$ \\
\hline 5.000 & 0.00224 & 0.01118 & 84 & 0.005 \\
2.000 & 0.00141 & 0.00283 & 130 & 0.024 \\
1.000 & 0.00100 & 0.00100 & 164 & 0.053 \\
0.500 & 0.00071 & 0.00035 & 182 & 0.076 \\
0.200 & 0.00045 & 0.000089 & 182 & 0.076 \\
0.100 & 0.00032 & 0.000032 & 182 & 0.076 \\
\hline
\end{tabular}

4.2. Dépôt au-dessus d'une crème de vase avec une concentration préalable faible Ce cas peut se présenter quand les vitesses du courant sont en train d'augmenter. Si les taux de dépôt préalables ont été élevés, la concentration à la surface de la crème de vase a pu diminuer jusqu'à sa valeur théorique minimale $\left(C_{S}=40 \mathrm{~kg} \mathrm{~m}^{-3}\right)$. La vase en cours de dépôt ne peut pas avoir une concentration supérieure à celle-ci en raison du tassement entravé.

Pour six valeurs de $C_{o}$, la figure 4 montre le taux de dépôt réduit $D^{e f} /\left(\overline{W_{o}} C_{o}\right)$ en fonction de $\tau_{o}$, calculé suivant l'approche retenue dans cette étude pour une crème de vase ayant une concentration en surface spécifique fixée arbitrairement à $75 \mathrm{~kg} \mathrm{~m}^{-3}$. Le tableau 2 récapitule plusieurs paramètres propres à cette modélisation.

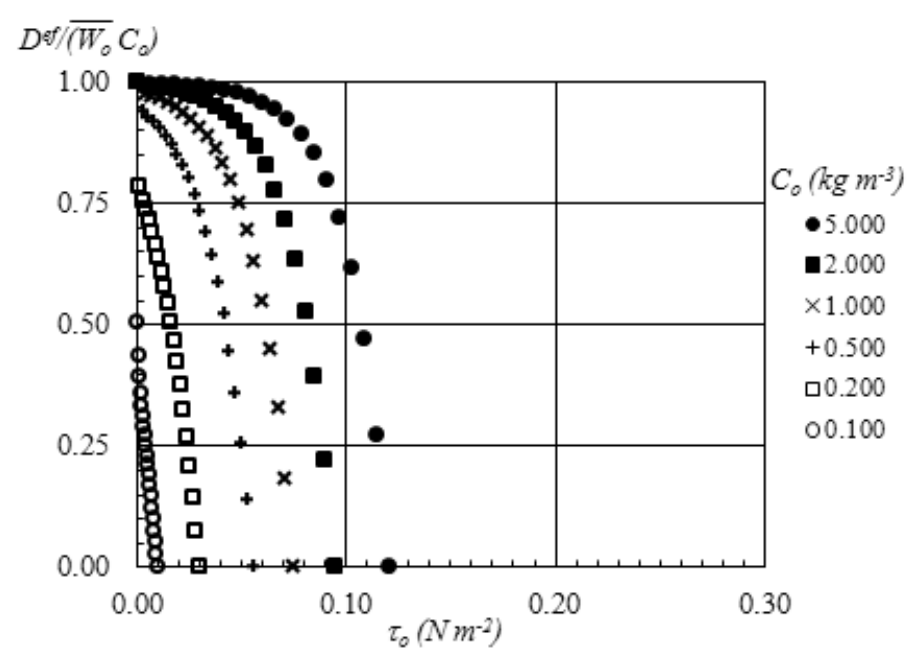

Figure 4. Valeurs de $D^{e f} /\left(\overline{W_{o}} C_{o}\right)$ simulées en fonction de $\tau_{o}$ pour un dépôt au-dessus d'une couche de crème de vase ayant une concentration $C_{S}=75 \mathrm{~kg} \mathrm{~m}^{-3}$ (concentration faible). 


\section{XII ${ }^{\text {èmes }}$ Journées Nationales Génie Côtier - Génie Civil \\ Cherbourg, 12-14 juin 2012}

Tableau 2. Paramètres de la modélisation de $D^{e f}$ pour une concentration faible de la vase préalablement déposée.

\begin{tabular}{lllll}
\hline$C_{o}\left(\mathrm{~kg} \mathrm{~m}^{-3}\right)$ & $\overline{W_{o}}\left(\mathrm{~m} \mathrm{~s}^{-1}\right)$ & $C_{o} \times \overline{W_{o}}\left(\mathrm{~kg} \mathrm{~m}^{-2} \mathrm{~s}^{-1}\right)$ & $C_{S}\left(\mathrm{~kg} \mathrm{~m}^{-3}\right)$ & $\tau_{e}\left(\mathrm{~N} \mathrm{~m}^{-2}\right)$ \\
\hline 5.000 & 0.00224 & 0.01118 & 75 & 0.0035 \\
2.000 & 0.00141 & 0.00283 & 75 & 0.0035 \\
1.000 & 0.00100 & 0.00100 & 75 & 0.0035 \\
0.500 & 0.00071 & 0.00035 & 75 & 0.0035 \\
0.200 & 0.00045 & 0.000089 & 75 & 0.0035 \\
0.100 & 0.00032 & 0.000032 & 75 & 0.0035 \\
\hline
\end{tabular}

\section{Conclusions}

Une modélisation phénoménologique du taux de dépôt effectif a été mise en œuvre sans utiliser explicitement le concept de Krone de valeur seuil $\tau_{d}$ de $\tau_{o}$ au-dessous de laquelle les sédiments se déposent (voir équation 1). Les taux de dépôts évalués sont proches de ceux calculés avec le modèle de Krone avec les valeurs usuelles de $\tau_{d}$. Cette étude ne met pas en cause la validité du modèle de Krone, mais constitue une première tentative pour comprendre les paramètres physiques régissant la valeur de $\tau_{d}$.

Il est vraisemblable que l'adhérence au fond des agrégats de vase entraînés par le flux de dépôt dépend de $C_{o}$ mais aussi de la concentration en surface des dépôts préalablement déposés. Ainsi, selon l'approche développée dans cette étude la valeur de $\tau_{d}$ semblent obéir aux lois de tendance suivantes qui restent à explorer et à valider par des observations expérimentales :

a) quand le dépôt se produit sur une couche de vase de concentration en surface élevée, la valeur de $\tau_{d}$ pourrait augmenter avec $C_{o}$ tant que la valeur de $C_{S}$ ne diminue pas en raison d'une forte valeur du flux de dépôt ;

b) quand le dépôt se produit sur une couche de crème de vase de très faible concentration en surface, la valeur de $\tau_{d}$ pourrait augmenter avec $C_{o}$ (voir figure 4).

Enfin, on peut noter que la variation linéaire de $D^{e f}$ selon le modèle de Krone n'est pas toujours satisfaisante et il est possible que dans certains cas une meilleure description du dépôt puisse être obtenue avec :

$D^{e f} \approx\left\{\begin{array}{lll}\overline{W_{o}} C_{o}, \text { si }: & \tau_{o}<\tau_{d} \\ 0, & \text { si : } & \tau_{o} \geq \tau_{d}\end{array}\right.$

\section{Références bibliographiques}

BEEN K. (1980). Stress-strain behaviour of a cohesive soil deposited under water. Ph.D. Thesis, Univ. Oxford, United Kingdom.

CERCO C.F., BUNCH B.W., TEETER A.M., DORTCH M.S. (2000). Water Quality Model of Florida Bay. U.S. Army Engineer Research and Development Center, ERDC/EL TR-00-10, Vicksburg, USA, 260 p. 
DELO E.A., OCKENDEN M.C. (1992). Estuarine Muds Manual. HR Wallingford Report SR309, 23 p.

HOSSEINI K. (1999). Liaison entre la rigidité initiale et la cohésion dans les vases molles - Relation avec la dynamique sédimentaire. Thèse, Université de Nantes, France. KRISHNAPPAN B.G., ENGEL P. (1994). Critical shear stresses for erosion and deposition of fine suspended sediments in Fraser River. National Water Research Institute, Burlington, Ontario, Canada, 10 p.

KRONE R.B. (1986). The significance of aggregate properties to transport processes. In: MEHTA A.J. (Ed.), Estuarine Cohesive Sediment Dynamics, Coastal and estuarine studies Vol. 14, Springer, Berlin, pp 66-84. doi:10.1007/978-1-4612-4936-8_4

KYNCH G.J. (1952). A theory of sedimentation. Faraday Society Transactions, Vol. 48, pp 166-176. doi:10.1039/tf9524800166

MEHTA A.J. (1986). Characterization of cohesive sediment properties and transport processes in estuaries. In: MEHTA A.J. (Ed.), Estuarine Cohesive Sediment Dynamics, Coastal and estuarine studies Vol. 14, Springer, Berlin, pp 290-325. doi:10.1007/978-1-46124936-8_15

MEHTA A.J., PARTHENIADES E. (1982). Resuspension of deposited cohesive sediment beds. Proceedings 18th Coastal Engineering Conference, Vol. 2, Cape Town, South Africa, pp 1569-1588.

MIGNIOT C. (1989). Tassement et rhéologie des vases. La Houille Blanche, Vol. 1 \& Vol. 2, pp 11-29 \& pp 95-111. doi:10.1051//hb/1989001 \& doi:10.1051/lhb/1989006

ROUSE H. (1937). Modern conceptions of the mechanics of fluid turbulence. Transactions of the American Society of Civil Engineers, Vol. 102, pp 463-541.

SANCHEZ M. (2006). Settling velocity of the suspended sediment in three high-energy environments. Ocean Engineering, Vol. 33, pp 665-678. doi:10.1016/j.oceaneng.2005.05.009

SANCHEZ M., GROVEL A. (1993). Modélisation du tassement sous poids propre des couches de vase molle et saturée sur un sol imperméable. La Houille Blanche, Vol. 1, pp 29-34. doi:10.1051//hb/1993002

SANCHEZ M., LEVACHER D. (2007). The influence of particle size of the dispersed mineral fraction on the settlement of marine and estuarine muds. Geo-Marine Letters, Vol. 27, pp 303-313. doi:10.1007/s00367-007-0053-7

SANCHEZ M., LEVACHER D. (2008). Erosion d'une vase de l'estuaire de la Loire sous l'action du courant. Bulletin of Engineering Geology and the Environment, Vol. 67, pp 597-605. doi:10.1007/s10064-008-0159-9

TEETER A.M. (1986). Vertical transport in fine-grained suspension and newlydeposited sediment. In: MEHTA A.J. (Ed.), Estuarine Cohesive Sediment Dynamics, Coastal and estuarine studies Vol. 14, Springer, Berlin, pp 170-191. doi:10.1007/978-1-46124936-8_9 